\title{
A stochastic framework for subspace identification of a strongly nonlinear aerospace structure
}

\author{
J.P. Noël ${ }^{1}$, J. Schoukens ${ }^{2}$, G. Kerschen ${ }^{1}$ \\ ${ }^{1}$ Space Structures and Systems Laboratory (S3L) \\ Department of Aerospace and Mechanical Engineering \\ University of Liège, Liège, Belgium \\ jp.noel, g.kerschen@ulg.ac.be \\ ${ }^{2}$ Department ELEC \\ Vrije Universiteit Brussel, Brussels, Belgium \\ johan.schoukens@vub.ac.be
}

\begin{abstract}
The present study exploits the maximum likelihood identification framework for deriving statistically-optimal models of nonlinear mechanical systems. The identification problem is formulated in the frequency domain, and model parameters are calculated by minimising a weighted least-squares cost function. Initial values of the model parameters are obtained by means of a nonlinear subspace algorithm. The complete identification methodology is first demonstrated on a Duffing oscillator, prior to being applied to a full-scale aerospace structure.
\end{abstract}

Keywords: System identification; maximum likelihood; subspace method; aerospace structure; nonsmooth nonlinearities.

\section{INTRODUCTION}

Even if nonlinear behaviour is known to be a frequent occurrence in structural dynamics ${ }^{[1-3]}$, at least in certain regimes of motion, common practice in industry is still to ignore nonlinearity. It has however become obvious in recent years that, to satisfy the continual interest in expanding the performance envelope of engineering systems, nonlinear components should be dealt with, or may even be constructively exploited, in the design process. This is why the development of effective system identification techniques applicable to nonlinear systems is today an active research area in the structural dynamics community ${ }^{[4]}$. In this context, the recently-introduced frequency-domain nonlinear subspace identification (FNSI) method ${ }^{[5]}$ is an interesting approach because it benefits from the numerical robustness and efficacy of subspace algorithms, while maintaining an acceptable computational burden. Promising applications of the FNSI method to low- and high-dimensional nonlinear systems from numerical and experimental data are reported in Refs. ${ }^{[5-7]}$.

The major disadvantage of subspace identification techniques is that they derive estimates of the model parameters based on deterministic arguments, and so do not provide any guarantee that the estimates still behave well in the presence of disturbing noise. An alternative approach to the identification problem is to embed it in a stochastic framework through the minimisation of a well-chosen cost function incorporating noise information ${ }^{[8]}$. In particular, the maximum likelihood cost function is particularly attractive because it yields estimates of the model parameters with optimal stochastic properties. Moreover, by formulating the cost function in the frequency domain, it is known to simplify to a weighted least-squares estimator. However, the maximum likelihood suffers from issues typically arisen in optimisation problems, especially related to initialisation. 
The contribution of the present work lies in the utilisation of the model parameter estimates provided by the FNSI method to serve as starting values for the minimisation of the maximum likelihood cost function. This initialisation strategy possesses the important advantage that the FNSI method generates a fully nonlinear model of the system under test, while classical approaches commonly use a linear model of the nonlinear system as starting point ${ }^{[9]}$. This ensures that the resulting maximum likelihood model performs at least as good as the nonlinear FNSI model. A second advantage is that the FNSI framework offers a convenient way to select an appropriate order for the nonlinear model, specifically using stabilisation diagrams, as described in Ref. ${ }^{[6]}$. The paper is organised as follows. Section 2 presents the model equations governing the vibrations of nonlinear systems. Sections 3 and 4 give a condensed introduction to the theoretical background of the FNSI method and maximum likelihood estimator, respectively. The proposed identification methodology is then demonstrated on a single-degree-of-freedom Duffing oscillator in Section 5. Section 6 finally investigates the applicability of the methodology to a full-scale aerospace structure possessing nonsmooth nonlinearities.

\section{NONLINEAR MODEL EQUATIONS AND PROBLEM STATEMENT}

The vibrations of nonlinear mechanical systems possessing an underlying linear regime of motion are governed by the timecontinuous model

$$
\mathbf{M} \ddot{\mathbf{q}}(t)+\mathbf{C}_{v} \dot{\mathbf{q}}(t)+\mathbf{K} \mathbf{q}(t)+\mathbf{g}(\mathbf{q}(t), \dot{\mathbf{q}}(t))=\mathbf{p}(t)
$$

where $\mathbf{M}, \mathbf{C}_{v}, \mathbf{K} \in \mathbb{R}^{n_{p} \times n_{p}}$ are the linear mass, viscous damping and stiffness matrices, respectively; $\mathbf{q}(t)$ and $\mathbf{p}(t) \in \mathbb{R}^{n_{p}}$ are the generalised displacement and external force vectors, respectively; $\mathbf{g}(t) \in \mathbb{R}^{n_{p}}$ is the nonlinear restoring force vector encompassing elastic and dissipative effects, and $n_{p}$ is the number of degrees of freedom (DOFs) of the structure obtained after spatial discretisation. The amplitude, direction, location and frequency content of the excitation $\mathbf{p}(t)$ determine in which regime the structure behaves.

The effects of the $r$ lumped nonlinear components in the system are represented using a linear-in-the-parameters model of the form

$$
\mathbf{g}(\mathbf{q}(t), \dot{\mathbf{q}}(t))=\sum_{a=1}^{r} \sum_{b=1}^{s_{a}} c_{a, b} \mathbf{h}_{a, b}(\mathbf{q}(t), \dot{\mathbf{q}}(t)) .
$$

In this double sum, $s_{a}$ is the number of nonlinear basis functions $\mathbf{h}_{a, b}(\mathbf{q}(t), \dot{\mathbf{q}}(t))$ selected to describe the $a$-th nonlinearity, and $c_{a, b}$ are the associated coefficients. The total number of nonlinear basis functions introduced in the model is equal to $s=\sum_{a=1}^{r} s_{a}$.

Given measurements of $\mathbf{p}(t)$ and $\mathbf{q}(t)$ or its derivatives, and an appropriate user selection of the functionals $\mathbf{h}_{a, b}(t)$, the identification problem addressed in the present paper aims at computing estimates of the parameters of a state-space model describing the dynamics governed by Eq. (1).

\section{GENERATING INITIAL PARAMETER ESTIMATES USING A NONLINEAR SUBSPACE IDENTIFICATION METHOD IN THE FREQUENCY DOMAIN}

The FNSI method is a subspace identification algorithm dedicated to mechanical system models incorporating linear-in-theparameters nonlinearities ${ }^{[5]}$. Linearity in the parameters avoids an iterative optimisation process, and issues related to initialisation and convergence thereof. The technique exploits data in the frequency domain and is naturally a multi-input, multi-output identification scheme as it constructs state-space models of nonlinear mechanical systems. Its implementation relies on robust tools from numerical analysis, including QR and singular value decompositions.

\subsection{Feedback interpretation of nonlinear structural dynamics and state-space model}

The FNSI methodology builds on a block-oriented interpretation of nonlinear structural dynamics, which sees nonlinearities as a feedback into the linear system in the open loop ${ }^{[10]}$, as illustrated in Fig. 1. This interpretation boils down to moving the nonlinear internal forces in Eq. (1) to the right-hand side, and viewing them as additional external forces applied to the underlying linear structure, that is

$$
\mathbf{M} \ddot{\mathbf{q}}(t)+\mathbf{C}_{v} \dot{\mathbf{q}}(t)+\mathbf{K} \mathbf{q}(t)=\mathbf{p}(t)-\sum_{a=1}^{r} \sum_{b=1}^{s_{a}} c_{a, b} \mathbf{h}_{a, b}(\mathbf{q}(t), \dot{\mathbf{q}}(t)) .
$$




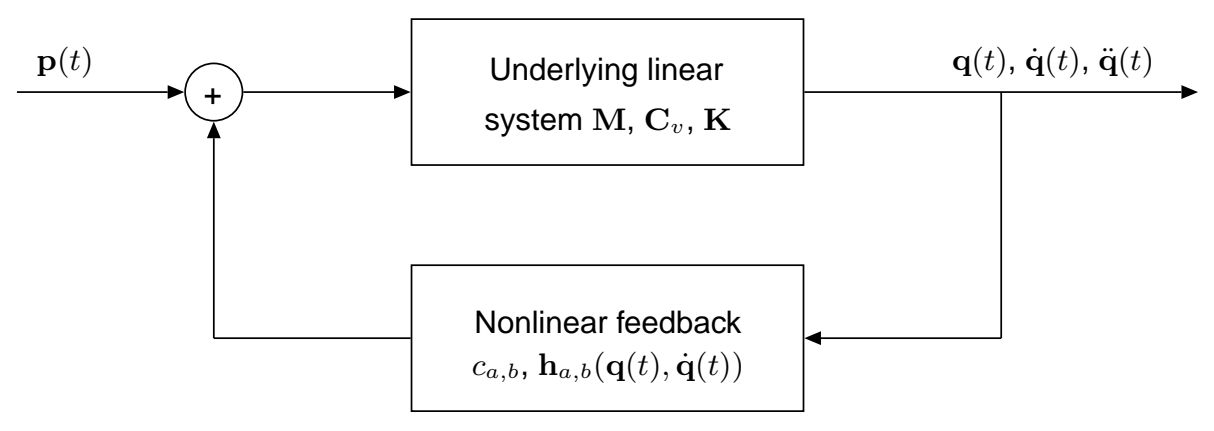

Figure 1: Feedback interpretation of nonlinear structural dynamics ${ }^{[10]}$.

Assuming that displacements are measured and defining the state vector $\mathbf{x}=\left(\mathbf{q}^{T} \dot{\mathbf{q}}^{T}\right)^{T} \in \mathbb{R}^{n_{s}}$, Eq. (3) is recast in the state space as the set of first-order equations

$$
\left\{\begin{aligned}
\dot{\mathbf{x}}(t) & =\mathbf{A}_{c} \mathbf{x}(t)+\mathbf{B}_{c} \mathbf{e}\left(\mathbf{p}(t), \mathbf{h}_{a, b}(t)\right) \\
\mathbf{q}(t) & =\mathbf{C}_{c} \mathbf{x}(t)+\mathbf{D}_{c} \mathbf{e}\left(\mathbf{p}(t), \mathbf{h}_{a, b}(t)\right)
\end{aligned}\right.
$$

where subscript $c$ stands for continuous-time, and where the vector $\mathbf{e} \in \mathbb{R}^{(s+1) n_{p}}$, termed extended input vector, concatenates the external forces $\mathbf{p}(t)$ and the nonlinear basis functions $\mathbf{h}_{a, b}(t)$. The matrices $\mathbf{A}_{c} \in \mathbb{R}^{n_{s} \times n_{s}}, \mathbf{B}_{c} \in \mathbb{R}^{n_{s} \times(s+1) n_{p}}, \mathbf{C}_{c} \in \mathbb{R}^{n_{p} \times n_{s}}$ and $\mathbf{D}_{c} \in \mathbb{R}^{n_{p} \times(s+1) n_{p}}$ are the state, extended input, output and direct feedthrough matrices, respectively. The dimension of the state space is $n_{s}=2 n_{p}$. State-space and physical-space matrices correspond through the relations

$$
\begin{gathered}
\mathbf{A}_{c}=\left(\begin{array}{cc}
\mathbf{0}^{n_{p} \times n_{p}} & \mathbf{I}^{n_{p} \times n_{p}} \\
-\mathbf{M}^{-1} \mathbf{K} & -\mathbf{M}^{-1} \mathbf{C}_{v}
\end{array}\right) \quad \mathbf{B}_{c}=\left(\begin{array}{ccccc}
\mathbf{0}^{n_{p} \times n_{p}} & \mathbf{0}^{n_{p} \times n_{p}} & \mathbf{0}^{n_{p} \times n_{p}} & \ldots & \mathbf{0}^{n_{p} \times n_{p}} \\
\mathbf{M}^{-1} & -c_{1,1} \mathbf{M}^{-1} & -c_{1,2} \mathbf{M}^{-1} & \ldots & -c_{r, s_{r}} \mathbf{M}^{-1}
\end{array}\right) \\
\mathbf{C}_{c}=\left(\begin{array}{lll}
\mathbf{I}^{n_{p} \times n_{p}} & \mathbf{0}^{n_{p} \times n_{p}}
\end{array}\right) \quad \mathbf{D}_{c}=\mathbf{0}^{n_{p} \times(s+1) n_{p}}
\end{gathered}
$$

where $\mathbf{0}$ and $\mathbf{I}$ are the zero and identity matrices, respectively. In a standard measurement setup, only limited sets of DOFs in $\mathbf{p}(t)$ and $\mathbf{q}(t)$ are excited and observed, respectively. The identification problem is therefore preferably stated in terms of $l$ applied forces and $m$ measured displacements collected in the vectors $\mathbf{u}(t) \in \mathbb{R}^{m \leq n_{p}}$ and $\mathbf{y}(t) \in \mathbb{R}^{l \leq n_{p}}$, respectively. Accordingly, the nonlinear basis functions vector is formed as $\mathbf{h}_{a, b}(\mathbf{y}(t), \dot{\mathbf{y}}(t))$, and the extended input vector is $\mathbf{e}\left(\mathbf{u}(t), \mathbf{h}_{a, b}(t)\right) \in \mathbb{R}^{m+s l}$. Eqs. (4) become

$$
\left\{\begin{aligned}
\dot{\mathbf{x}}(t) & =\mathbf{A}_{c} \mathbf{x}(t)+\mathbf{B}_{c} \mathbf{e}\left(\mathbf{u}(t), \mathbf{h}_{a, b}(t)\right) \\
\mathbf{y}(t) & =\mathbf{C}_{c} \mathbf{x}(t)+\mathbf{D}_{c} \mathbf{e}\left(\mathbf{u}(t), \mathbf{h}_{a, b}(t)\right)
\end{aligned}\right.
$$

where $\mathbf{A}_{c}, \mathbf{B}_{c}, \mathbf{C}_{c}$ and $\mathbf{D}_{c}$ are now projections of the original matrices onto the controlled and observed DOFs. Note that no identifiability condition constraints the number of measured displacements, provided that the nonlinear basis functions $\mathbf{h}_{a, b}(t)$ can be formed from the recorded channels. In particular, $l$ can be lower than the model order $n_{s}$, as will be demonstrated in Section 6 . In practice, the number of instrumented locations is generally dictated by the spatial resolution of the structural mode shapes the user wants to obtain in order to limit linear modelling errors, and by the number of available sensors.

Although there is a full equivalence between time- and frequency-domain identification ${ }^{[8]}$, differences may arise in the way acquired information is formulated in the two domains. In particular, experimental data are commonly recorded as frequency responses, power spectral densities or merely discrete Fourier transform (DFT) spectra, which are all more compact than timedomain data and, in turn, substantially decrease the computational burden. Moreover, frequency data provide an intuitive understanding of the nature and importance of nonlinear distortions in the dynamics of the system under test ${ }^{[11,12]}$. These arguments motivate the development of a nonlinear subspace methodology in the frequency domain.

It is however well-known that frequency-domain subspace algorithms formulated in continuous time are badly conditioned and generally lead to poor estimates. This is due to the particular structure of the data matrices introduced in subspace identification ${ }^{[5]}$. Solutions exist to improve the conditioning of the inverse problem in the continuous-time domain, such as the use of the Forsythe recursions ${ }^{[13]}$ or the w-operator ${ }^{[14]}$. Nevertheless, we prefer to guarantee the good conditioning of the proposed algorithm by means of a discrete-time transformation of Eqs. (6), before applying the DFT. Eqs. (6) eventually write

$$
\left\{\begin{array}{r}
z_{f} \mathbf{X}(f)=\mathbf{A}_{d} \mathbf{X}(f)+\mathbf{B}_{d} \mathbf{E}(f) \\
\mathbf{Y}(f)=\mathbf{C}_{d} \mathbf{X}(f)+\mathbf{D}_{d} \mathbf{E}(f)
\end{array}\right.
$$


where subscript $d$ stands for discrete-time, and where $z_{f}=e^{j 2 \pi f / N}$ is the Z-transform variable, and $\mathbf{X}(f), \mathbf{E}(f)$ and $\mathbf{Y}(f)$ are the DFTs of $\mathbf{x}(t), \mathbf{e}\left(\mathbf{u}(t), \mathbf{h}_{a, b}(t)\right)$ and $\mathbf{y}(t)$, respectively. A classical frequency-domain subspace resolution scheme can finally be applied to Eqs. (7) in order to identify the four discrete-time system matrices $\mathbf{A}_{d}, \mathbf{B}_{d}, \mathbf{C}_{d}$ and $\mathbf{D}_{d}$. This application basically involves the reformulation of Eqs. (7) in matrix form, and the computation of estimates of the matrices through appropriate geometrical manipulations of input and output data. The interested reader is referred to Ref. ${ }^{[5]}$ for a detailed introduction to the theoretical and practical aspects of the FNSI method.

\subsection{Conversion from discrete-time state space to continuous-time physical space}

The estimated discrete-time model $\left(\widehat{\mathbf{A}_{d}}, \widehat{\mathbf{B}_{d}}, \widehat{\mathbf{C}_{d}}, \widehat{\mathbf{D}_{d}}\right)$ is first converted into the continuous-time domain ${ }^{[5]}$, where the underlying linear modal properties and the nonlinear coefficients $c_{a, b}$ of the system can next be estimated. To achieve the transformation back to physical space, Eq. (2) is substituted into Eq. (1) in the frequency domain to yield

$$
\mathbf{G}^{-1}(\omega) \mathbf{Q}(\omega)+\sum_{a=1}^{r} \sum_{b=1}^{s_{a}} c_{a, b} \mathbf{H}_{a, b}(\omega)=\mathbf{P}(\omega)
$$

where $\mathbf{G}(\omega)$ is the FRF matrix of the underlying linear system, and where $\mathbf{Q}(\omega), \mathbf{H}_{a, b}(\omega)$ and $\mathbf{P}(\omega)$ are the Fourier transforms of $\mathbf{q}(t), \mathbf{h}_{a, b}(t)$ and $\mathbf{p}(t)$, respectively. The concatenation of $\mathbf{P}(\omega)$ and $\mathbf{H}_{a, b}(\omega)$ further introduces the extended input spectrum $\mathbf{E}(\omega)$, so as to obtain the linear relationship between $\mathbf{Q}(\omega)$ and $\mathbf{E}(\omega)$

$$
\mathbf{Q}(\omega)=\mathbf{G}(\omega)\left[\begin{array}{llll}
\mathbf{I}^{n_{p} \times n_{p}} & -c_{1,1} \mathbf{I}^{n_{p} \times n_{p}} & \ldots & -c_{r, s_{r}} \mathbf{I}^{n_{p} \times n_{p}}
\end{array}\right] \mathbf{E}(\omega)=\mathbf{G}^{e}(\omega) \mathbf{E}(\omega) .
$$

Matrix $\mathbf{G}^{e}(\omega)$, termed extended FRF matrix, encompasses the underlying linear FRF matrix of the system and the nonlinear coefficients. Moreover, Ref. ${ }^{[15]}$ proved that it is an invariant system property that can be calculated, similarly to linear theory, from the combination of the continuous-time state-space matrices as

$$
\mathbf{G}^{e}(\omega)=\widehat{\mathbf{C}_{c}}\left(j \omega \mathbf{I}^{n_{s} \times n_{s}}-\widehat{\mathbf{A}_{c}}\right)^{-1} \widehat{\mathbf{B}_{c}}+\widehat{\mathbf{D}_{c}}
$$

As a result, the nonlinear coefficients identified from $\mathbf{G}^{e}(\omega)$ using Eqs. (9) and (10) are spectral quantities, i.e. they are complexvalued and frequency-dependent. This is an attractive property, because the importance of the frequency variations and imaginary parts of the coefficients is particularly convenient for assessing the quality of the identification results. This possibility relies on the fact that, in the noise-free case and in the absence of modelling errors, the real parts of the coefficients converge (for an infinite number of measured samples) to their exact values with no frequency dependence, and the imaginary parts converge to zero. A reliable identification scheme together with an appropriate selection of the nonlinear functionals $\mathbf{h}_{a, b}(t)$ should therefore make the imaginary parts much smaller than the corresponding real parts. The frequency dependence of the coefficients should also remain small. An extensive analysis of the link between linear and nonlinear modelling errors and the frequency dependence and imaginary parts of the nonlinear coefficients can be found in Ref. ${ }^{[6]}$.

\section{OPTIMISING INITIAL PARAMETER ESTIMATES IN THE MAXIMUM LIKELIHOOD FRAMEWORK}

The FNSI algorithm presented in Section 3 belongs to a family of identification methods relying on a deterministic approach. Specifically, assuming that the noiseless input and output measurements obey specific model equations, namely the Newton's law of dynamics given in Eq. (1), the model parameters are retrieved by manipulating these equations by means of geometrical operations. The major disadvantage of deriving an estimation technique based on deterministic arguments is that the user has no guarantee that it behaves well in the presence of disturbing noise.

By contrast, a systematic approach to building an estimator in a stochastic framework is to formulate it as the minimiser of a cost function, expressing the distance in some metric between model's predicted outputs and system's measured outputs ${ }^{[8,16]}$. The cost function usually incorporates knowledge about the disturbing noise to ensure that the resulting estimator has a reliable noise behaviour. In this context, the maximum likelihood cost function is particularly attractive because it yields estimates of the model parameters with optimal stochastic properties, namely consistency and efficiency ${ }^{[8]}$. Loosely speaking, consistency translates that the estimates converge to their true vales for an infinite number of measured samples. These estimates also exhibit the lowest possible uncertainty given the signal-to-noise ratio, which is referred to as the efficiency property of the maximum likelihood estimator (MLE).

The price to pay for consistency and efficiency is the knowledge of the probability density function of the noise in the formulation of the maximum likelihood cost function. This is, in practice, a hard requirement to deal with. However, by applying the DFT to 
input and output data, the distribution of noise can be proved to be Gaussian, with a predominantly-diagonal covariance matrix, regardless its time-domain distribution ${ }^{[17]}$. This implies that the MLE boils down to a weighted least-squares estimator in the frequency domain. Introducing the vector of model parameters

$$
\theta=\left[\operatorname{vec}\left(\widehat{\mathbf{A}_{c}}\right) ; \operatorname{vec}\left(\widehat{\mathbf{B}_{c}}\right) ; \operatorname{vec}\left(\widehat{\mathbf{C}_{c}}\right) ; \operatorname{vec}\left(\widehat{\mathbf{D}_{c}}\right)\right]
$$

where the vector operation denoted vec stacks the columns of a matrix on top of each other, the cost function $\mathbf{V}$ to minimise hence writes

$$
\mathbf{V}(\theta)=\sum_{f=1}^{F} \epsilon^{H}(f, \theta) \mathbf{W}(f) \epsilon(f, \theta)
$$

where $H$ denotes the conjugate transpose of a matrix, and $F$ is the number of frequency lines exploited in the identification. The model error vector $\epsilon(f, \theta) \in \mathbb{C}^{l}$ is defined as the complex-valued difference

$$
\epsilon(f, \theta)=\mathbf{Y}_{m}(f, \theta)-\mathbf{Y}(f)
$$

where $\mathbf{Y}_{m}(f, \theta)$ and $\mathbf{Y}(f)$ are the DFTs of the modelled outputs in Eqs. (6) and the measured outputs, respectively. Furthermore, the matrix $\mathbf{W}(f) \in \mathbb{C}^{l \times l}$ introduced in Eq. (12) discriminates between good and poor frequency measurements through a proper weighting. It is typically chosen as the inverse of the covariance matrix of the outputs, which can be easily extracted directly from recorded data if a periodic excitation signal is employed.

\section{DEMONSTRATION ON A SINGLE-DEGREE-OF-FREEDOM DUFFING OSCILLATOR}

In this section, the identification methodology proposed in Sections 3 and 4 is demonstrated using a single-degree-of-freedom system with one nonlinearity, namely a Duffing oscillator. The vibrations of the Duffing oscillator are governed by the equation

$$
M \ddot{q}(t)+C_{v} \dot{q}(t)+K q(t)+c_{1,1} q^{3}(t)=p(t) .
$$

The linear and nonlinear parameters selected in this analysis are listed in Table 1. The system was simulated using a nonlinear Newmark time integration scheme, with a sampling frequency of $15000 \mathrm{~Hz}$. Simulated time series were then decimated down to $750 \mathrm{~Hz}$ for practical use, considering low-pass filtering to avoid aliasing. The excitation $p(t)$ was a random phase multisine ${ }^{[8]}$. A random phase multisine is a periodic random signal with an user-controlled amplitude spectrum. If an integer number of periods is measured, this spectrum is perfectly realised, unlike classical Gaussian noise. The main advantage of a multisine is that its periodic nature can be exploited to separate signal from noise, what leads to an easy estimation of the noise covariance matrix. A multisine with a flat amplitude spectrum in $5-150 \mathrm{~Hz}$ was chosen herein, thus encompassing the third harmonics of the oscillator around $110 \mathrm{~Hz}$. The root-mean-squared (RMS) amplitude of the excitation was set to $100 \mathrm{~N}$.

\begin{tabular}{cccc}
\hline$M(k g)$ & $C_{v}(N s / m)$ & $K(k N / m)$ & $c_{1,1}\left(M N / m^{3}\right)$ \\
2 & 10 & 100 & 100 \\
\hline \multicolumn{2}{c}{ Natural frequency $\omega(H z)$} & \multicolumn{2}{c}{ Damping ratio $\epsilon(\%)$} \\
35.59 & \multicolumn{2}{c}{1.12} \\
\hline
\end{tabular}

TABLE 1: Parameters of the Duffing oscillator.

The time simulation was conducted over 30 periods of 8192 samples, and Gaussian white noise was added to the synthetic signals to recreate the disturbances observed in a real measurement setup. The noise level was set to $2 \%$ of the RMS amplitude of the response. The first 5 periods of measurement were rejected to settle the transients, and the last 5 periods were saved for validation purposes. Measurements were averaged over the remaining 20 periods to mitigate noise and obtain a sample estimate of its covariance matrix. Throughout the paper, it is assumed that noise corrupts the system's outputs $\mathbf{q}(t)$ only, whereas the nonlinear basis functions $\mathbf{h}_{a, b}(\mathbf{q}(t), \dot{\mathbf{q}}(t))$ are observed without errors. This is a simplifying assumption because the basis functions are typically formed in practice using displacements and velocities obtained by integrating noisy accelerometer signals. One exception that should yet be pointed out is the direct measurement of the displacements at the nonlinearity locations, using techniques such as scanning laser Doppler vibrometers ${ }^{[18,19]}$ which provide very large signal-to-noise ratios.

The first step of the identification methodology is the application of the FNSI method to obtain initial estimates of the parameters of a state-space model of the system. In the case of the Duffing, this model comprises 12 parameters, i.e. all the elements of the matrices $\mathbf{A}_{c}, \mathbf{B}_{c}, \mathbf{C}_{c}$ and $\mathbf{D}_{c}$ in Eqs. (6), given 2 states, 1 input, 1 nonlinear basis function and 1 output. The 12 state-space 


\begin{tabular}{lccc}
\hline & Error on $\omega(\%)$ & Error on $\epsilon(\%)$ & Error on $c_{1,1}(\%)$ \\
FNSI & $-1010^{-4}$ & -0.13 & 0.71 \\
MLE & $410^{-4}$ & -0.02 & 1.29 \\
\hline
\end{tabular}

TABLE 2: Relative errors on the estimation of the natural frequency $\omega$, damping ratio $\epsilon$ and nonlinear coefficient $c_{1,1}$ of the Duffing oscillator using the FNSI method (first row) and the MLE (second row).

parameters can be converted into estimates of the natural frequency, damping ratio and nonlinear coefficient of the oscillator, as explained in Section 3.2. They are given in the first row of Table 2 through relative errors, and are found to be in good agreement with their reference values.

However, these 3 parameters do not convey a thorough assessment of the accuracy of the subspace model. A sounder basis for this assessment is the direct comparison of the measured and reconstructed output spectra of the system, as achieved in Fig. 2. This figure reveals that the prediction of the subspace model suffers from important modelling errors, in particular in the vicinity of resonance. A similar conclusion is drawn from the analysis of the corresponding time series presented in Fig. 3, where the RMS value of the FNSI error is equal to $1.68 \mathrm{~mm}$, compared to the RMS amplitude of the response of $4.25 \mathrm{~mm}$.

The modelling errors of the subspace model can be significantly reduced by minimising the maximum likelihood objective function in Eq. (12). The resulting error on the prediction of the output spectrum is showed after 10 iterations in Fig. 2 using blue circles. Modelling errors have now an amplitude comparable to the noise level. Note, however, that the improvements brought to the estimation of the parameters $\omega, \epsilon$ and $c_{1,1}$ remain marginal (see Table 2). The error of the maximum likelihood model is also plotted in the time domain in Fig. 3, and corresponds to a RMS value of $0.02 \mathrm{~mm}$. The good behaviour of the MLE is finally confirmed in Fig. 4, where the prediction errors of both subspace and maximum likelihood models are computed on validation data.

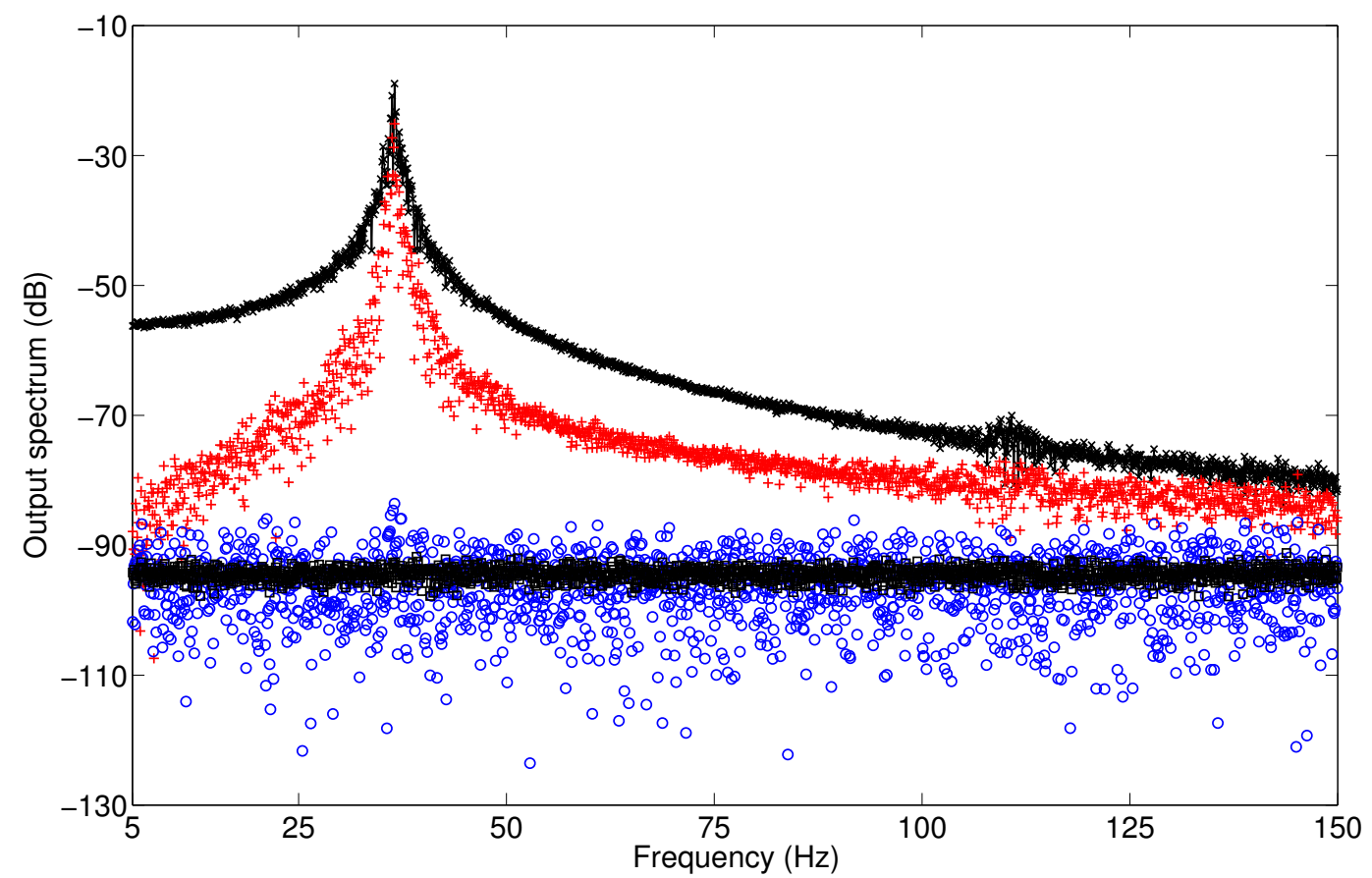

Figure 2: Output spectrum of the Duffing oscillator. Black cross: measured spectrum averaged over 20 periods; red plus: error of the FNSI model; blue circle: error of the MLE model; black square: noise level. 


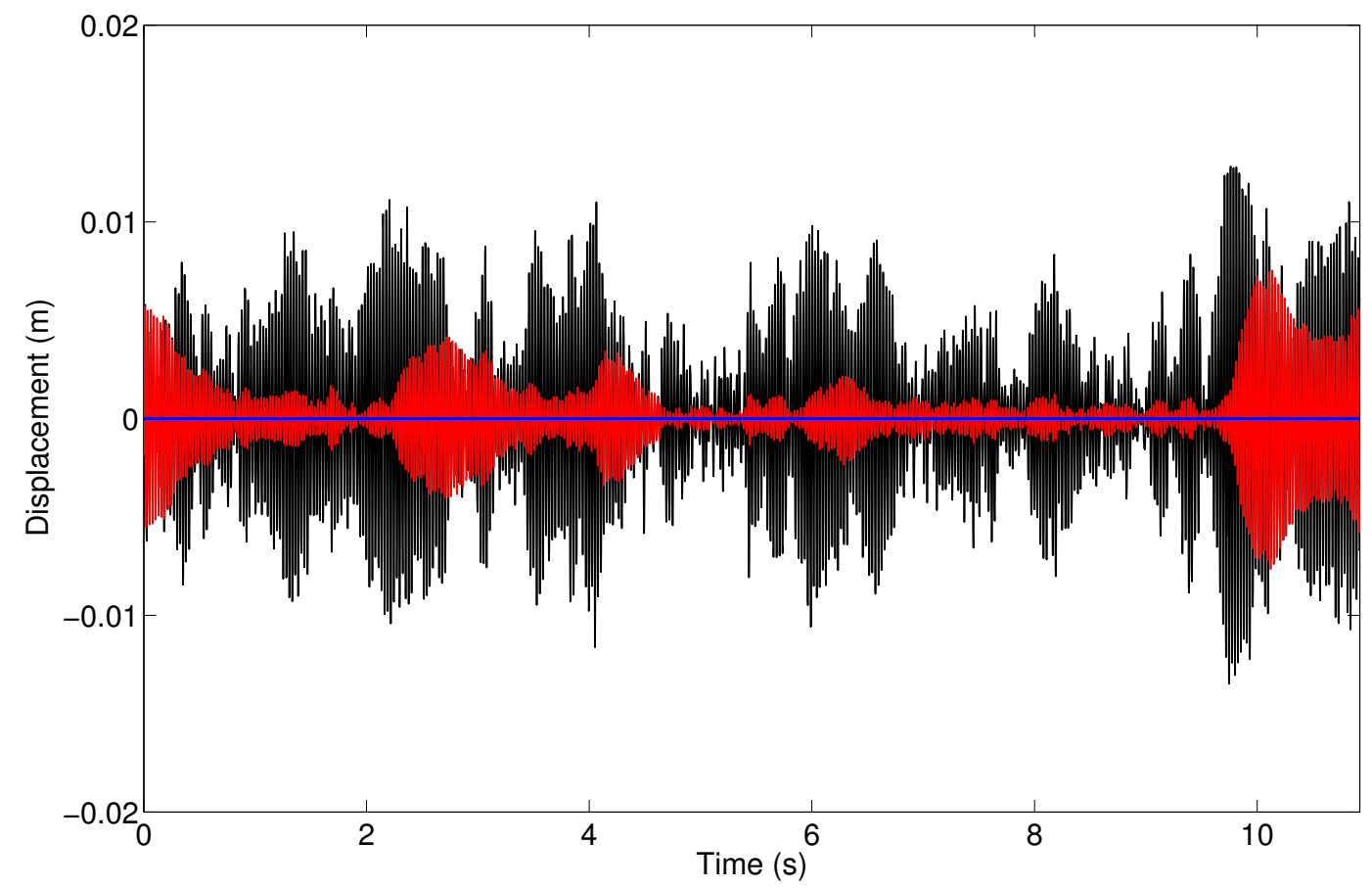

Figure 3: Time response of the Duffing oscillator. Black: measured response averaged over 20 periods; red: error of the FNSI model; blue: error of the MLE model.

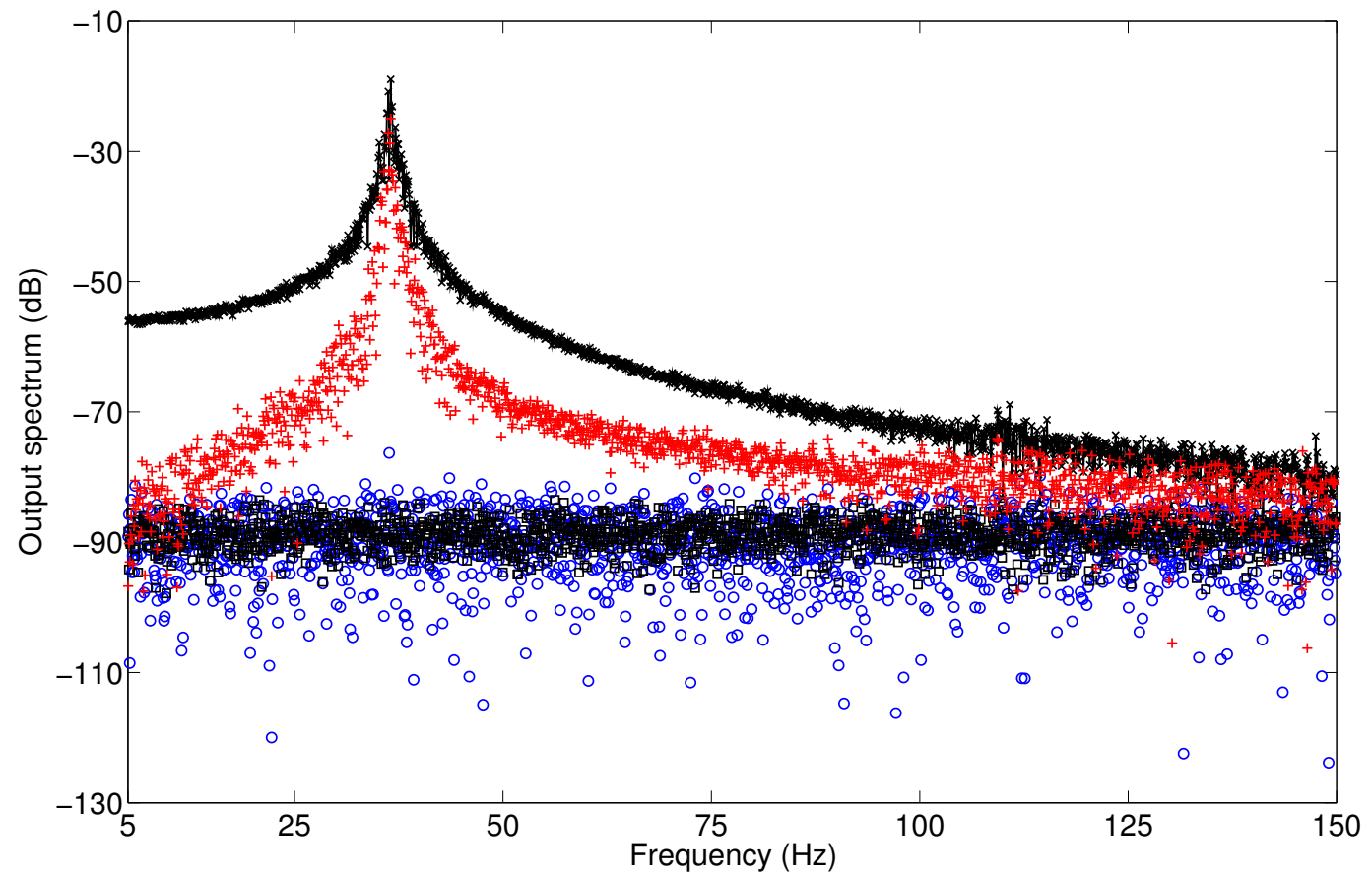

Figure 4: Validation of the subspace and maximum likelihood models. Black cross: measured output spectrum averaged over 5 periods; red plus: error of the FNSI model; blue circle: error of the MLE model; black square: noise level. 


\section{APPLICATION TO A FULL-SCALE STRONGLY NONLINEAR SATELLITE STRUCTURE}

This section investigates the applicability of the proposed identification methodology in the case of a full-scale aerospace structure possessing nonsmooth nonlinearities, the SmallSat spacecraft developed by EADS-Astrium.

\subsection{Description and modelling of the SmallSat spacecraft}

The SmallSat structure was conceived by EADS-Astrium as a low-cost platform for small satellites in low earth orbits ${ }^{[20]}$. It is a monocoque tube structure which is $1.2 \mathrm{~m}$ in height and $1 \mathrm{~m}$ in width. It is composed of eight flat faces for equipment mounting purposes, creating an octagon shape, as shown in Figure 5 . The octagon is manufactured using carbon-fibre-reinforced plastic by means of a filament winding process. The structure thickness is $4 \mathrm{~mm}$ with an additional $0.25-\mathrm{mm}$-thick skin of Kevlar applied to both the inside and outside surfaces to provide protection against debris. The top floor is an $1-m^{2}$ sandwich aluminium panel, with $25-\mathrm{mm}$ core and $1-\mathrm{mm}$ skins. The interface between the spacecraft and launch vehicle is achieved via four aluminium brackets located around cut-outs at the base of the structure. The total mass including the interface brackets is around $64 \mathrm{~kg}$.

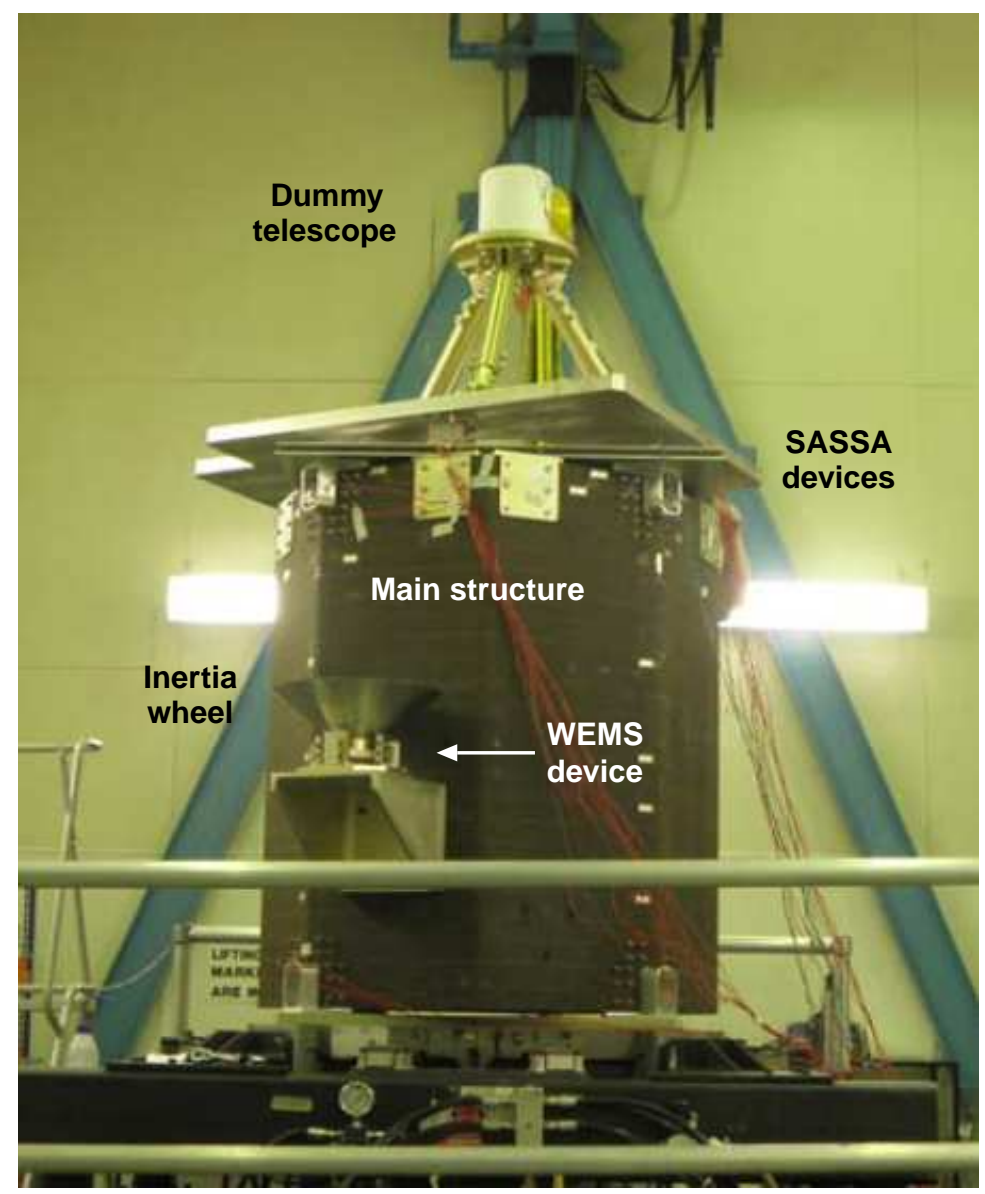

Figure 5: SmallSat spacecraft equipped with an inertia wheel supported by the WEMS device and a dummy telescope connected to the main structure by the SASSA isolators.

The spacecraft structure supports a dummy telescope mounted on a baseplate through a tripod; its mass is around $140 \mathrm{~kg}$. The dummy telescope plate is connected to the SmallSat top floor by three shock attenuators, termed shock attenuation system for spacecraft and adaptor (SASSA) ${ }^{[21]}$, whose behaviour is considered as linear in this study. Besides, as depicted in Figure 6 (a), a support bracket connects to one of the eight walls the so-called wheel elastomer mounting system (WEMS) device which is loaded with an 8- $\mathrm{kg}$ dummy inertia wheel. The WEMS device acts as a mechanical filter which mitigates high-frequency 
disturbances coming from the inertia wheel through the presence of a soft elastomeric interface between its mobile part, i.e. the inertia wheel and a supporting metallic cross, and its fixed part, i.e. the bracket and by extension the spacecraft. Moreover, the WEMS incorporates eight mechanical stops, covered with a thin layer of elastomer, and designed to limit the axial and lateral motions of the inertia wheel during launch, which gives rise to strongly nonlinear dynamical phenomena.

Figure 6 (b) presents a simplified, yet relevant, modelling of the WEMS device where the inertia wheel, owing to its important rigidity, is seen as a point mass. The four nonlinear connections (NCs) between the WEMS mobile and fixed parts are labelled NC $1-4$. Each NC possesses a trilinear spring in the axial direction (elastomer in traction/compression plus two stops), a bilinear spring in the radial direction (elastomer in shear plus one stop) and a linear spring in the third direction (elastomer in shear). In Figure 6 (b), linear and nonlinear springs are denoted by squares and circles, respectively.
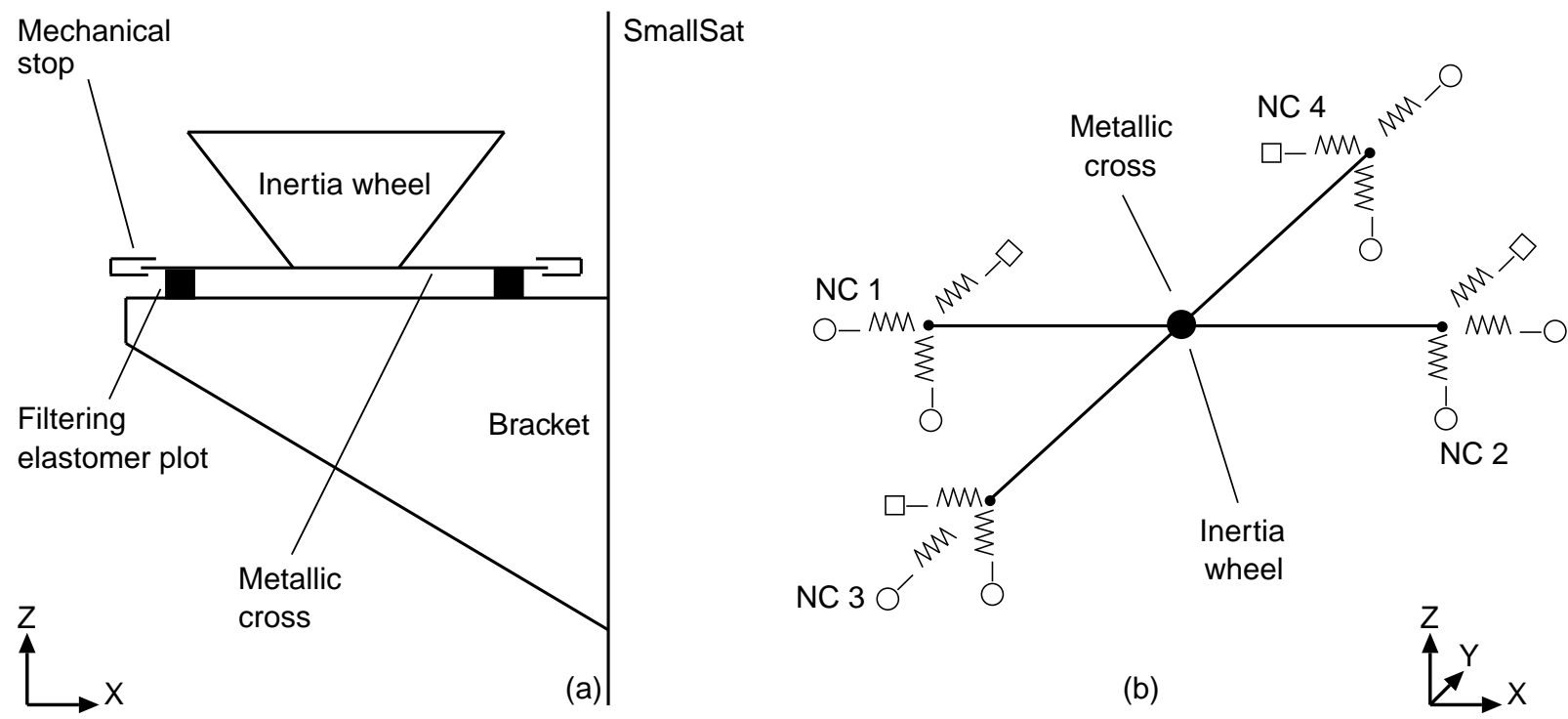

Figure 6: WEMS device. (a) Detailed description of the WEMS components; (b) simplified modelling of the WEMS mobile part considering the inertia wheel as a point mass. The linear and nonlinear connections between the WEMS mobile and fixed parts are signalled by squares and circles, respectively.

A finite element model (FEM) of the SmallSat was developed in Samcef software and used in the present work to conduct numerical experiments. It comprises about 65000 DOFs and the comparison with experimental data revealed its good predictive capabilities. The model consists of shell elements (octagon structure and top floor, instrument baseplate, bracket and WEMS metallic cross) and point masses (dummy inertia wheel and telescope) and meets boundary conditions with four clamped nodes. Proportional damping is considered and the high dissipation in the elastomer components of the WEMS is described using lumped dashpots, hence resulting in a highly non-proportional damping matrix. Table 3 gives the natural frequencies $\omega$ and damping ratios $\epsilon$ of the first 11 modes of the SmallSat up to $50 \mathrm{~Hz}$, together with a brief description of the corresponding deformations of the structure. One remarks that the WEMS local modes, which are likely to involve nonlinear dynamics, are clustered in two groups around 9 and $23 \mathrm{~Hz}$. Subsequent resonances involve bracket bending and axial and lateral motions of the telescope supporting panel.

To achieve tractable nonlinear calculations, the linear elements of the FEM were condensed using the Craig-Bampton reduction technique. This approach consists in expressing the system dynamics in terms of some retained DOFs and internal modes of vibration. Specifically, the full-scale model of the spacecraft was reduced to 13 nodes (excluding DOFs in rotation), namely both sides of each NC (8), the inertia wheel point mass (1) and the four corners of the instrument baseplate (4), and 100 internal modes. In total, the reduced-order model thus contains 139 DOFs. Piecewise-linear springs were finally introduced within the WEMS module between the NC nodes considering clearances and stiffness parameters identified experimentally ${ }^{[22]}$ and listed in Table 4. To avoid numerical issues, third-order polynomials were utilised in the close vicinity of the clearances to implement $C^{1}$ continuity. 


\begin{tabular}{|c|c|c|c|}
\hline Mode & $\begin{array}{c}\text { Natural } \\
\text { frequency } \omega(H z)\end{array}$ & $\begin{array}{l}\text { Damping } \\
\text { ratio } \epsilon(\%)\end{array}$ & Description \\
\hline 1 & 8.55 & 10.77 & $\begin{array}{l}\text { Concave motion of the } \\
\text { WEMS mobile part (WMP) along } X\end{array}$ \\
\hline 2 & 8.97 & 11.09 & Symmetric to mode 1 along $Y$ \\
\hline 3 & 18.17 & 24.55 & Rotation of the WMP around Z \\
\hline 4 & 22.31 & 12.14 & Convex motion of the WMP along $\mathrm{X}$ \\
\hline 5 & 23.17 & 11.31 & Symmetric to mode 4 along $Y$ \\
\hline 6 & 31.57 & 5.46 & $\begin{array}{l}\text { Bracket bending and } \\
\text { in-phase motion of the WMP along Z }\end{array}$ \\
\hline 7 & 31.59 & 2.10 & $\begin{array}{l}\text { Bracket bending and } \\
\text { rotation of the instrument panel (IP) }\end{array}$ \\
\hline 8 & 32.66 & 2.57 & $\begin{array}{l}\text { Bracket bending and } \\
\text { rotation of the IP perpendicularly to mode } 7\end{array}$ \\
\hline 9 & 37.47 & 2.27 & $\begin{array}{c}\text { Rotation of the IP similarly to mode } 8 \\
\text { and rotation around } Z\end{array}$ \\
\hline 10 & 38.25 & 2.33 & $\begin{array}{c}\text { Main structure bending and in-plane motion } \\
\text { of the IP in phase }\end{array}$ \\
\hline 11 & 43.31 & 2.51 & Rotation of the IP similarly to mode 8 \\
\hline
\end{tabular}

TABLE 3: Natural frequencies $\omega$ and damping ratios $\epsilon$ of the first 11 modes of the SmallSat up to $50 \mathrm{~Hz}$ and description of the corresponding deformations of the structure.

\begin{tabular}{|c|c|c|c|c|c|}
\hline & Neg. clearance & Pos. clearance & Linear stiffness & Neg. nonlinear stiffness & Pos. nonlinear stiffness \\
\hline X1 & 1.90 & - & 0.70 & 26.76 & - \\
\hline X2 & - & 1.93 & 0.75 & - & 46.23 \\
\hline X3 & - & - & 0.77 & - & - \\
\hline X4 & - & - & 0.55 & - & - \\
\hline Y1 & - & - & 0.82 & - & - \\
\hline Y2 & - & - & 0.70 & - & - \\
\hline Y3 & 1.90 & - & 0.58 & 26.76 & - \\
\hline Y4 & - & 1.93 & 1.02 & - & 46.23 \\
\hline Z1 & 1.01 & 1.55 & 8.30 & 118.07 & 79.40 \\
\hline Z2 & 0.84 & 1.62 & 9.21 & 116.73 & 88.41 \\
\hline Z3 & 0.93 & 1.59 & 9.18 & 118.07 & 79.40 \\
\hline Z4 & 0.93 & 1.59 & 10.03 & 116.73 & 88.41 \\
\hline
\end{tabular}

TABLE 4: Experimental clearances and stiffness parameters of the WEMS piecewise-linear springs given through adimensional values for confidentiality reasons. 


\subsection{Activation of a single nonlinearity of the WEMS device}

A multisine with a flat amplitude spectrum in $5-50 \mathrm{~Hz}$ was applied in the axial direction to NC 2 on the inertia wheel side. Time integration was carried out over 30 periods of 8192 samples using a nonlinear Newmark scheme considering a sampling frequency of $20000 \mathrm{~Hz}$. Time series were subsequently decimated down to $1000 \mathrm{~Hz}$. The amplitude and the location of the excitation caused axial impacts exclusively in NC 2. The level of Gaussian noise added to the synthetic signals was set to $2 \%$ of the RMS amplitude of the axial response at the inertia wheel node. Similarly to the analysis of the Duffing oscillator in Section 5, 3 periods were rejected to avoid transient distortions, and 5 periods were kept for validating the subspace and maximum likelihood models. The average of the 22 remaning periods eventually yielded an estimate of the covariance matrix of noise corrupting each channel.

The first step toward formulating a nonlinear subspace model of the SmallSat dynamics is the selection of an adequate model order, which translates the number of linear modes excited in the output data ${ }^{[5]}$. In linear system identification, stabilisation diagrams are most frequently exploited as decision-making tools and have proved successful in numerous industrial applications. A distinct advantage of the FNSI method is that it still allows the use of the stabilisation diagram for retrieving linear system parameters from nonlinear data ${ }^{[6]}$. Fig. 7 charts the stabilisation of the natural frequencies, damping ratios and mode shapes of the structure for model orders up to 60. This analysis was conducted using only three output sensors, namely the axial DOFs of both nodes of NC 2 and of the inertia wheel node. This corresponds to the practical situation where the number of available channels is limited and where system's responses are only recorded close to the nonlinearity. Note also that the modal assurance criterion for complex-valued mode shapes (MACX), as defined in reference ${ }^{[23]}$, is utilised in this diagram as the damping mechanisms in the SmallSat were shown to be highly non-proportional. Fig. 7 shows full stabilisation of 4 modes at order 10. However, since this is tested between two successive model orders taking as reference the lowest order, equal stabilisation is also achieved at order 8 , which is therefore selected to avoid spurious poles.

The genuine poles captured at order 8 correspond to modes 1, 4, 6 and 8 of the structure. Table 5 lists the relative errors on the estimates of their natural frequencies and damping ratios and the diagonal MACX values between the identified and exact mode shapes. The selection of order 8 results in a state-space model with 121 parameters, given 8 states, 1 input, 2 nonlinear basis functions and 3 outputs. The recourse to two nonlinear basis functions translates the activation of the negative and positive axial mechanical stops at NC 2. The associated coefficients are depicted in Fig. 8 using black lines and are listed in Table 6 through the averaged values of their real parts, relative errors and ratios between their real and imaginary parts.

\begin{tabular}{|c|c|c|c|c|c|c|}
\hline \multirow[t]{2}{*}{ Mode } & \multicolumn{2}{|c|}{ Error on $\omega(\%)$} & \multicolumn{2}{|c|}{ Error on $\epsilon(\%)$} & \multicolumn{2}{|c|}{ MACX } \\
\hline & FNSI & MLE & FNSI & MLE & FNSI & MLE \\
\hline 1 & -0.17 & -0.16 & -0.54 & -0.62 & 1.00 & 1.00 \\
\hline 4 & 0.05 & 0.06 & -0.64 & -0.62 & 1.00 & 1.00 \\
\hline 6 & -0.20 & -0.18 & -6.31 & -6.38 & 1.00 & 1.00 \\
\hline 8 & 0.07 & 0.07 & 13.69 & 13.84 & 1.00 & 1.00 \\
\hline
\end{tabular}

TABLE 5: Relative errors on the estimated linear natural frequencies and damping ratios (in \%) and diagonal MACX values computed by the FNSI method and the MLE using three measured channels.

\begin{tabular}{lcccccc}
\hline & \multicolumn{2}{c}{ Real part } & \multicolumn{2}{c}{ Error (\%) } & \multicolumn{2}{c}{$\log _{10}($ Real/Imag.) } \\
& FNSI & MLE & FNSI & MLE & FNSI & MLE \\
& & & & & & \\
Neg. nonlinear coefficient & 117.80 & 100.54 & 0.92 & -13.87 & 3.05 & 1.09 \\
Pos. nonlinear coefficient & 88.74 & 102.63 & 0.37 & 16.09 & 1.95 & 1.83 \\
\hline
\end{tabular}

TABLE 6: Estimates of the negative and positive nonlinear stiffness coefficients at NC 2 computed by FNSI and the MLE. Real parts averaged over $5-50 \mathrm{~Hz}$, relative errors (in \%) and ratios between the real and imaginary parts (in logarithmic scaling). 


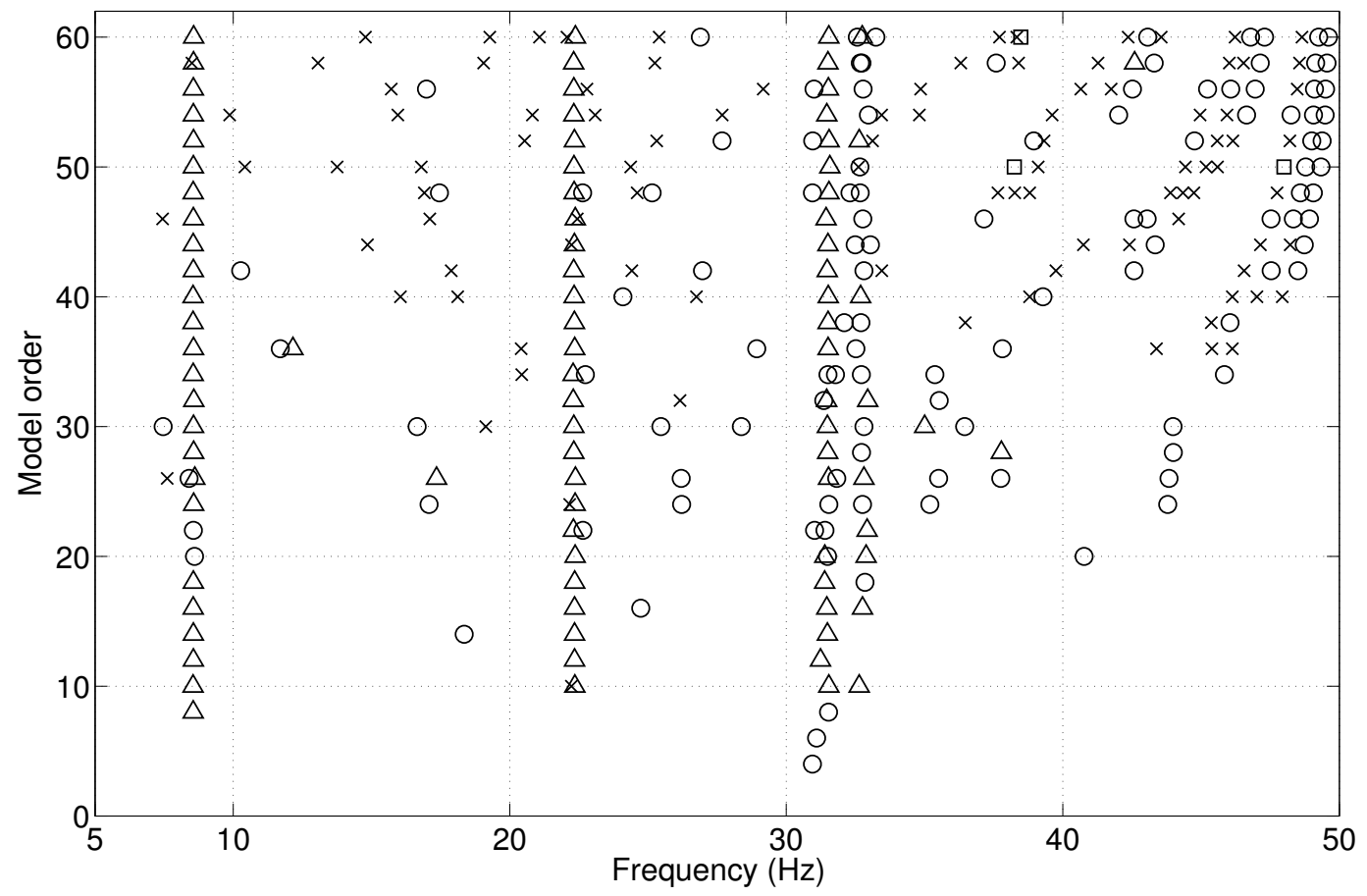

Figure 7: Stabilisation diagram computed by the FNSI method using three measured channels. Cross: stabilisation in natural frequency; square: extra stabilisation in damping ratio; circle: extra stabilisation in MACX; triangle: full stabilisation. Stabilisation thresholds in natural frequency, damping ratio and MACX value are $2 \%, 5 \%$ and 0.98 , respectively.

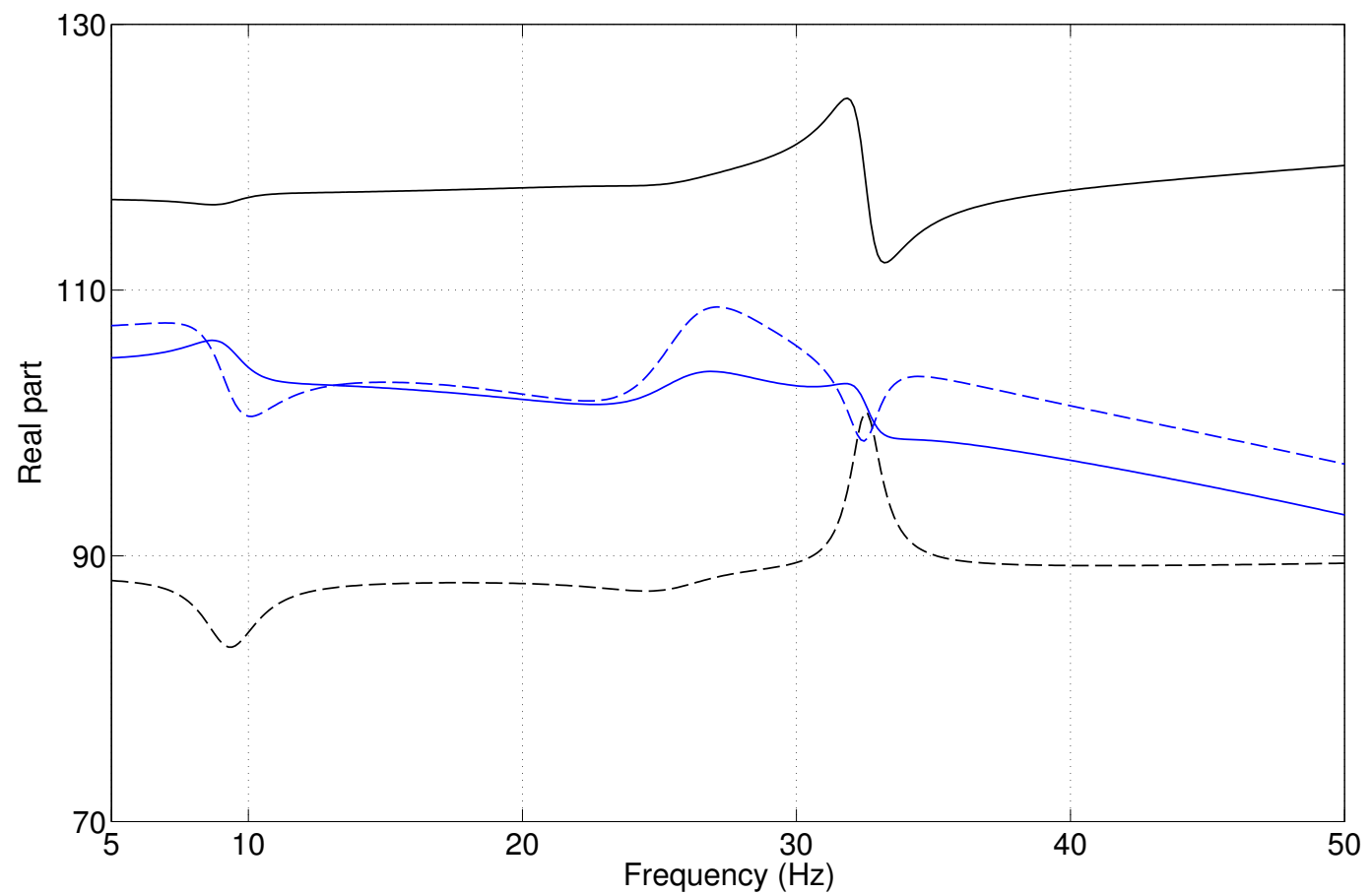

Figure 8: Real parts of the estimates of the negative (solid lines) and positive (dashed lines) nonlinear stiffness coefficients at NC 2 computed by FNSI (black lines) and the MLE (blue lines). 
Fig. 9 shows the comparison between the measured output spectrum at the inertia wheel in the $Z$ direction and the error of the corresponding spectrum predicted by the FNSI model. Important modelling errors can be observed, though being generally located $10 \mathrm{~dB}$ below the response level. The time responses associated with Fig. 9 are plotted in Fig. 10. The RMS value of the system's response is equal to $0.77 \mathrm{~mm}$, while the subspace modelling error is limited to a RMS value of $0.11 \mathrm{~mm}$. The application of maximum likelihood iterations to the initial subspace model leads to a clear decrease of the modelling errors, as evidenced in Fig. 9 through the blue circles and in Fig. 10 with the blue curve corresponding to a RMS value of $0.03 \mathrm{~mm}$. As in Section 5, the MLE yields no improvement of the estimates of the modal properties (see Table 5). One also notes in Table 6 and in Fig. 8 that it degrades the estimation of the nonlinear coefficients. Moreover, unlike the case of the Duffing oscillator, the modelling errors corresponding to the maximum likelihood model cannot be reduced down to the noise level. This inability to eliminate modelling errors completely is also clearly visible on validation data, as presented in Fig. 11. All this can be attributed to several factors, including linear modelling errors due to the limited number of processed channels, inaccuracies in the computation of the Jacobian matrix of the problem which was realised using central finite differences, and the presence of local minima in the weighted least-squares objective function.

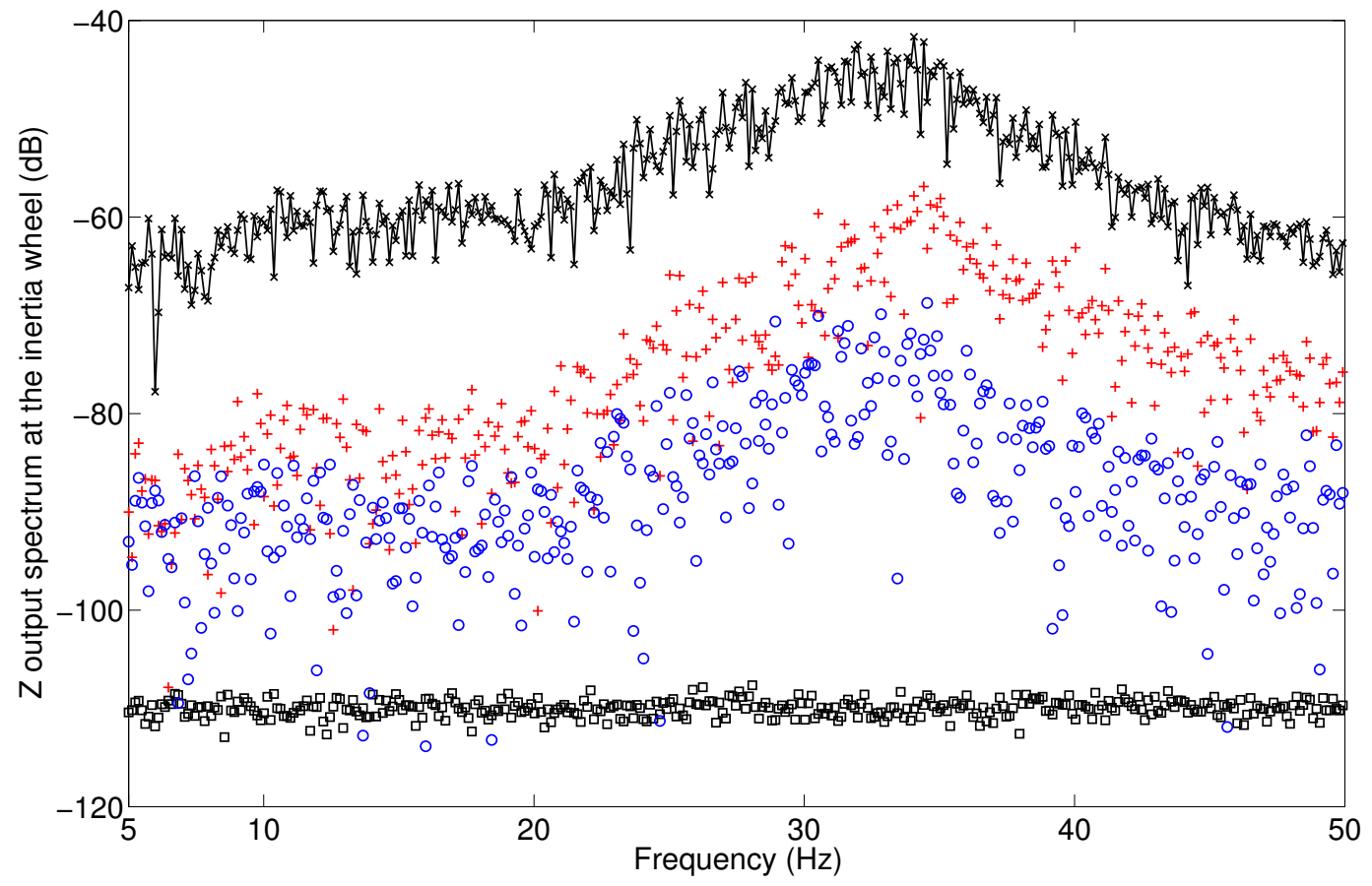

Figure 9: Output spectrum at the inertia wheel in the Z direction. Black cross: measured spectrum averaged over 23 periods; red plus: error of the FNSI model; blue circle: error of the MLE model; black square: noise level. 


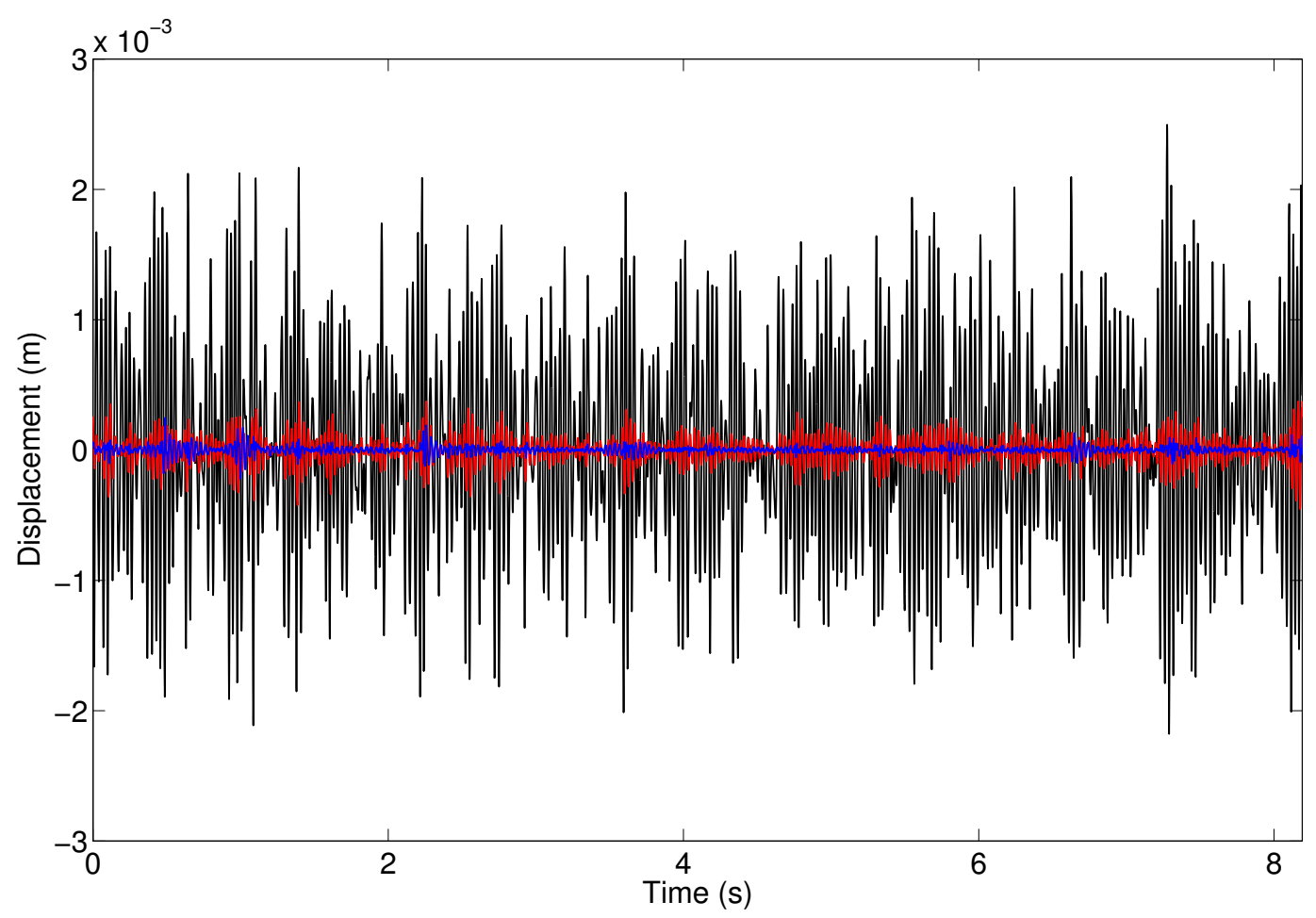

Figure 10: Time response at the inertia wheel in the $Z$ direction. Black: measured response averaged over 23 periods; red: error of the FNSI model; blue: error of the MLE model.

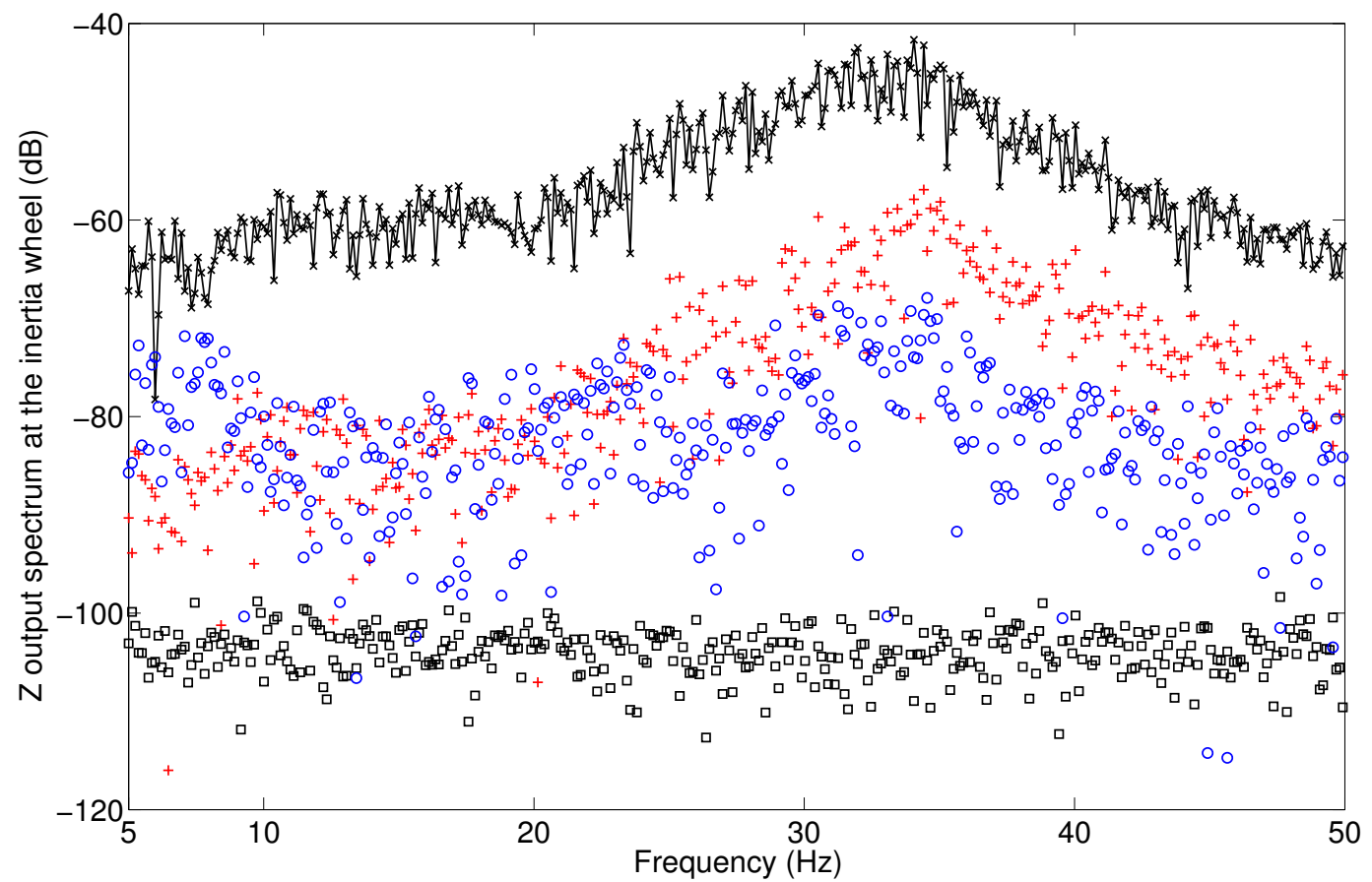

Figure 11: Validation of the subspace and maximum likelihood models at the inertia wheel in the Z direction. Black cross: measured output spectrum averaged over 5 periods; red plus: error of the FNSI model; blue circle: error of the MLE model; black square: noise level. 


\section{CONCLUSIONS}

The objective of the present paper was to combine the advantages of the subspace and maximum likelihood estimators to derive a stochastic identification framework dedicated to nonlinear mechanical systems. For that purpose, the recently-introduced FNSI method was employed to generate an initial state-space model of the system. A weighted least-squares objective function in the frequency domain was then minimised to obtain statistically-optimal parameter estimates. The methodology was successfully demonstrated on a Duffing oscillator. Its applicability was also investigated in the case of a large-scale satellite structure exhibiting strongly nonlinear dynamics. In this second application, significant reduction of the subspace modelling errors was attained, without however being able to eliminate them completely. Further work should therefore focus on the improvement of the maximum likelihood cost function through, e.g., a careful analysis of the linear modelling errors and an exact computation of the Jacobian matrix of the optimisation problem. A particular attention should also be devoted to this Jacobian matrix as it may lead to the calculation of uncertainty bounds for the model parameters.

\section{ACKNOWLEDGEMENTS}

The author J.P. Noël is a Research Fellow (FRIA fellowship) of the Fonds de la Recherche Scientifique - FNRS which is gratefully acknowledged.

\section{REFERENCES}

[1] Ahlquist, J. R., Carreno, J. M., Climent, H., de Diego, R. and de Alba, J., Assessment of nonlinear structural response in A400M GVT, Proceedings of the International Modal Analysis Conference XXVIII, 2010, Jacksonville, FL.

[2] Link, M., Boeswald, M., Laborde, S., Weiland, M. and Calvi, A., Non-linear experimental modal analysis and application to satellite vibration test data, Proceedings of the 3rd International Conference on Computational Methods in Structural Dynamcs and Earthquake Engineering, 2011, Corfu, Greece.

[3] Noël, J. P., Renson, L., Kerschen, G., Peeters, B., Manzato, S. and Debille, J., Nonlinear dynamics analysis of an F-16 aircraft using GVT data, Proceedings of the International Forum on Aeroelasticity and Structural Dynamics, 2013, Bristol, UK.

[4] Kerschen, G., Worden, K., Vakakis, A. F. and Golinval, J. C., Past, present and future of nonlinear system identification in structural dynamics, Mechanical Systems and Signal Processing, Vol. 20, pp. 505-592, 2006.

[5] Noël, J. P. and Kerschen, G., Frequency-domain subspace identification for nonlinear mechanical systems, Mechanical Systems and Signal Processing, Vol. 40, pp. 701-717, 2013.

[6] Noël, J. P., Marchesiello, S. and Kerschen, G., Subspace-based identification of a nonlinear spacecraft in the time and frequency domains, Mechanical Systems and Signal Processing, in press.

[7] Noël, J. P., Kerschen, G., Foltête, E. and Cogan, S., Frequency-domain subspace identification of nonlinear mechanical systems - Application to a solar array structure, Proceedings of the International Modal Analysis Conference XXXI, 2013, Garden Grove, CA.

[8] Pintelon, R. and Schoukens, J., System Identification: A Frequency Domain Approach, IEEE Press, New York, 1st edn., 2001.

[9] Paduart, J., Lauwers, L., Swevers, J., Smolders, K., Schoukens, J. and Pintelon, R., Identification of nonlinear systems using Polynomial Nonlinear State Space models, Automatica, Vol. 46, pp. 647-656, 2010.

[10] Adams, D. E. and Allemang, R. J., A frequency domain method for estimating the parameters of a non-linear structural dynamic model through feedback, Mechanical Systems and Signal Processing, Vol. 14, No. 4, pp. 637-656, 2000.

[11] Schetzen, M., The Volterra and Wiener Theories of Nonlinear Systems, Wiley, New York, 1st edn., 1980.

[12] Schoukens, J., Pintelon, R., Rolain, Y. and Dobrowiecki, T., Frequency response function measurements in the presence of nonlinear distortions, Automatica, Vol. 37, pp. 939-946, 2001. 
[13] Van Overschee, P. and De Moor, B., Continuous-time frequency domain subspace system identification, Signal Processing, Vol. 52, pp. 179-194, 1996.

[14] Yang, Z. J. and Sanada, S., Frequency domain subspace identification with the aid of the w-operator, Electrical Engineering in Japan, Vol. 132, No. 1, pp. 46-56, 2000.

[15] Marchesiello, S. and Garibaldi, L., A time domain approach for identifying nonlinear vibrating structures by subspace methods, Mechanical Systems and Signal Processing, Vol. 22, pp. 81-101, 2008.

[16] Ljung, L., System Identification: Theory for the User, Prentice-Hall, Upper Saddle River, 2nd edn., 1999.

[17] Schoukens, J. and Renneboog, J., Modeling of noise influence of the Fourier coefficients after a discrete Fourier transform, IEEE Transactions on Instrumentation and Measurement, Vol. 35, No. 3, pp. 278-286, 1986.

[18] Sracic, M. W., Allen, M. S. and Sumali, H., Identifying the modal properties of nonlinear structures using measured free response time histories from a scanning last Doppler vibrometer, Proceedings of the International Modal Analysis Conference XIX, 2012, Jacksonville, FL.

[19] Giuliani, P., Di Maio, D., Schwingshackl, C. W., Martarelli, M. and Ewins, D. J., Six degrees of freedom measurement with continuous scanning laser doppler vibrometer, Mechanical Systems and Signal Processing, Vol. 20, No. 2, pp. 367383, 2013.

[20] Russell, A. G., Thick skin, faceted, CFRP, monocoque tube structure for smallsats, Proceedings of the European Conference on Spacecraft Structures, Materials and Mechanical Testing, 2000, Noordwijk, The Netherlands.

[21] Camarasa, P. and Kiryenko, S., Shock attenuation system for spacecraft and adaptor (SASSA), Proceedings of the European Conference on Spacecraft Structures, Materials and Mechanical Testing, 2009, Toulouse, France.

[22] Noël, J. P., Renson, L. and Kerschen, G., Experimental identification of the complex dynamics of a strongly nonlinear spacecraft structure, Proceedings of the ASME International Design Engineering Technical Conferences, 2013, Portland, OR.

[23] Vacher, P., Jacquier, B. and Bucharles, A., Extension of the MAC criterion to complex modes, Proceedings of the International Conference on Noise and Vibration Engineering, 2010, Leuven, Belgium. 